\title{
Características Quantitativas das Partes do Corpo Não-Integrantes da Carcaça Animal e Desenvolvimento do Trato Gastrintestinal de Bezerros da Raça Holandesa Alimentados com Dietas Contendo Quatro Níveis de Concentrado
}

\section{Ricardo Dias Signoretti ${ }^{1}$, Gherman Garcia Leal de Araújo를 José Fernando Coelho da Silva ${ }^{3}$, Sebastião de Campos Valadares Filho ${ }^{3}$, Paulo Roberto Cecon ${ }^{4}$, Marcelo de Andrade Ferreira ${ }^{5}$}

\begin{abstract}
RESUMO - Trinta e dois bezerros da raça Holandesa, puros por cruzamento, variedade preto e branco, com, em média, 75 dias de idade e $78 \mathrm{~kg}$ PV inicial, divididos em dois grupos de PV de abate (grupo 1: 16 animais abatidos com $190 \pm 10 \mathrm{~kg}$ PV e grupo 2: 16 animais abatidos com $300 \pm 10 \mathrm{~kg}$ PV), foram distribuídos em delineamento inteiramente casualizado, para avaliar o efeito dos diferentes níveis de concentrado das dietas sobre as características quantitativas das partes do corpo não-integrantes da carcaça e o desenvolvimento do trato gastrintestinal (TGI). Os animais foram alimentados à vontade com dietas contendo 45,0; 60,0; 75,0; e 90,0\% de concentrado, na base da MS, usando-se feno de capim coast-cross (Cynodon dactylon), fubá de milho e farelo de soja, os quais constituíram dietas com aproximadamente $16 \%$ PB. A cada período de 28 dias, os animais de cada grupo foram pesados e abatidos, quando se aproximaram de PV preestabelecidos para os grupos 1 e 2. Não foram verificados efeitos dos níveis de concentrado nas dietas sobre os pesos, em valores absolutos e por $100 \mathrm{~kg}$ PCV, de rúmen-retículo, intestino delgado (ID), intestino grosso (IG), couro, gordura interna e comprimento do IG, para ambos os grupos. Para os animais do grupo 1, houve efeito quadrático sobre o peso do omaso, enquanto o peso do abomaso, em valores absolutos, para ambos os grupos, aumentou linearmente, em função dos níveis de concentrado nas dietas. O comprimento do ID, por 100kg PCV, dos animais de grupo 2, diminuiu linearmente com a adição de concentrado nas dietas. O peso de órgãos internos (MOI), em valores absolutos, dos animais do grupo 2, não foi influenciado, ao passo que o dos animais do grupo 1 foi influenciado pela adição de concentrado nas dietas. Os pesos da MOI, por $100 \mathrm{~kg}$ PCV, para os animais do grupo 1 , foram influenciados, porém o peso da gordura mesentérica, em valores absolutos e por $100 \mathrm{~kg}$ de PCV, para os animais do grupo 2 , elevou-se linearmente com aumento do nível de concentrado nas dietas.
\end{abstract}

Palavras-chave: abomaso, bezerros holandeses, concentrado, couro, intestino, mesentério, omaso, órgãos internos, rúmen-retículo, sangue

\section{Quantitative Traits of Parts of the Body Non Integrate of the Animal Carcass and Development of the Gastrointestinal Tract of Holsteins Calves Fed Diets with Four Concentrate Levels}

\begin{abstract}
Thirty two Holstein black and white type bull calves averaging 75 days of age and initial $78 \mathrm{~kg}$ LW, divided into two slaughter groups (group 1: 16 animals slaughtered with $190 \pm 10 \mathrm{~kg} \mathrm{LW}$ and group 2: 16 animals slaughtered with $300 \pm 10 \mathrm{~kg}$ LW) were allotted to a completely randomized design to evaluate the effects of crescent dietary levels of concentrate on the quantitative traits of the non integrate body parts of the animal carcass and the development of the gastrointestinal tract (GIT). The animals were full fed with diets contained 45.0, 60.0, 75.0 and $90.0 \%$ of concentrate on the DM basis, using coast-cross (Cynodon dactylon) grass hay and corn meal, and soybean meal, which constituted diets with approximately $16,0 \% \mathrm{CP}$. At each 28 days period, the animals from each group were weighed and slaughtered at preestablished weights for the groups 1 and 2 . The concentrate levels of the diet did not affect the weights, in absolute values and per $100 \mathrm{~kg}$ EBW, of reticulum-rumen (RR), small intestine (SI), large intestine (LI), hide, and internal fat and in the length of LI for both groups. For animals from the group 1, there was a quadratic effect on the omasum weight, while the abomasum weight, for both groups, linearly increased in function of concentrate levels in the diet. The length of SI, per $100 \mathrm{~kg} \mathrm{EBW}$, from animal of the group 2 , decreased linearly, with the addition of concentrate in the diet. The weights of internal organs (MIO), in absolute values, from animals of the group 2 were not affected. However, animals from the group 1 were affected by the addition of concentrate in the diets. The weight of MIO, per $100 \mathrm{~kg} \mathrm{EBW}$, for the animals from the group 1, were affected, but the mesenteric fat weight in absolute values and per $100 \mathrm{~kg}$ EBW, for animals from the group 2, linearly increased, with the crescent levels concentrate in diets.
\end{abstract}

Key Words: Abomasum, holstein calves, concentrate, hide, intestine, mesenteric, omasum, internal organs, reticulum-rumen, blood

\footnotetext{
${ }^{1}$ Eng.-Agr., Doutor em Zootecnia.

2 Pesquisador do CPATSA - EMBRAPA. 56300-000 Petrolina, PE.

3 Professor do DZO/UFV. Bolsista do CNPq - 36571-000 - Viçosa, MG.

${ }^{4}$ Professor do Departamento de Matemática da UFV.

5 Professor do Departamento de Zootecnia da UFRPE.
} 


\section{Introdução}

As funções primárias do trato gastrintestinal (TGI) e seus órgãos acessórios são a digestão e a absorção de nutrientes essenciais para os processos metabólicos. Todavia, poucas pesquisas têm sido feitas por parte dos nutricionistas de ruminantes sobre os aspectos quantitativos das partes não-integrantes da carcaça, pois estas tendem a variar de acordo com a raça, o estádio de maturidade e o nível nutricional, tendo, conseqüentemente, influência sobre o rendimento de carcaça, as exigências de mantença e o ganho de peso (GEAY, 1975).

Além disso, o consumo alimentar (JOHNSON et al., 1990; PERON et al., 1993) e a habilidade de digerir os alimentos (VALADARES FILHO, 1985) podem ser influenciadas em virtude de diferenças entre raças, estádio de maturidade e dieta fornecida aos animais. Essas diferenças podem estar relacionadas, em parte, às características anatômicas do TGI, como tamanho relativo das partes do trato digestivo, que sofrem alterações com o crescimento e desenvolvimento dos bovinos.

Segundo OLIVEIRA et al. (1992), os componentes do TGI tiveram maior desenvolvimento relativo, em animais mais leves, pois as vísceras atingem o desenvolvimento máximo mais precocemente que os tecidos ósseo e muscular.

$\mathrm{O}$ regime alimentar e o peso de abate exerceram influência sobre as proporções das diferentes partes do corpo não-integrantes da carcaça. $\mathrm{O}$ menor peso observado para TGI de animais em regime de consumo restrito sugere possível atrofia deste, em resposta ao baixo consumo de alimentos imposto durante a permanência dos animais no confinamento (PERON et al., 1993).

PERON et al. (1993) observaram que os animais da raça Holandesa apresentaram maior peso do TGI vazio (estômagos e intestinos vazios) que os de raças especializadas de corte, provavelmente, em virtude de a seleção ser direcionada principalmente para produção de leite, que exigiu maior consumo de alimentos, o que contribuiu para que esses animais apresentassem menores rendimentos de carcaça.

Os órgãos vitais e o esqueleto foram os componentes que apresentaram seu maior desenvolvimento numa fase mais precoce da vida do animal, ocasião em que a taxa de crescimento dos mesmos suplantou a do crescimento muscular e dos tecidos adiposos; situação que se inverteu com o crescimento do animal (GALVÃO et al., 1991).

O tamanho do fígado de novilhos respondeu rapidamente (três dias) às mudanças no consumo de alimentos, apresentando desenvolvimento linear crescente com aumento do consumo de energia metabolizável (RICHMOUD et al., 1988; JOHNSON et al., 1990).

CALIL (1978) citou que, em bovinos, o jejum de dois dias, antes do abate, pode resultar em perda de peso do fígado de até $25 \%$. Da mesma forma, HOGG (1991) afirmou que, quando o estresse nutricional induz manutenção ou perda de peso, há declínio no peso e na proporção dos órgãos internos, particularmente do fígado, dos estômagos e dos intestinos.

LUNT et al. (1986) verificaram que a raça, o ganho de peso diário e a dieta influíram na massa de órgãos internos (MOI), pois novilhos alimentados com dietas à base de volumosos apresentaram maiores pesos de coração e fígado que os alimentados à base de grãos.

Dentro deste contexto, as diferenças nos tamanhos relativos do TGI e da MOI, entre raças e estágio de maturidade, poderiam refletir em diferenças nas exigências de mantença (energia e proteína), pois principalmente o fígado, o coração, as glândulas mamárias e os tecidos do TGI, dentre todos os tecidos corporais, relacionaram-se entre os de maior atividade metabólica (FERREL et al., 1976; FERREL e JENKINS, 1983).

Maiores pesos de outras partes não componentes da carcaça, principalmente couro e peso conjunto de cabeça, pés e couro, expressas em \%PCVZ, tenderam a diminuir o rendimento de carcaça (GALVÃO et al., 1991; PERON et al., 1993; e JORGE et al., 1996).

Objetivou-se, neste estudo, verificar o desenvolvimento relativo (pesos e comprimentos) dos compartimentos do TGI, da MOI e das outras partes nãointegrantes da carcaça de bezerros da raça Holandesa submetidos a sistema de alimentação à vontade, em que as dietas continham $45,60,75$ e $90 \%$ de concentrado.

\section{Material e Métodos}

O experimento foi conduzido nas dependências do Laboratório Animal do Departamento de Zootecnia da Universidade Federal de Viçosa, Minas Gerais.

Foram usados 32 bezerros da raça Holandesa, puros por cruzamento, não-castrados, com idade média de 75 dias e peso vivo médio inicial de $78 \mathrm{~kg}$, alimentados ad libitum, sendo 16 para o grupo 1 ( $190 \pm 10 \mathrm{~kg}$ de peso vivo ao abate) e 16 para o grupo $2(300 \pm 10 \mathrm{~kg}$ de peso vivo ao abate). As dietas utilizadas foram constituídas de feno de capim coastcross (Cynodon dactylon), previamente picado, fubá de milho, farelo de soja, calcário e mistura mineral, formuladas para atender as exigências de energia e proteína, segundo as normas do NRC (1989), para 
Rev. bras. zootec.

ganho de peso vivo esperado de $1,0 \mathrm{~kg} / \mathrm{dia}$.

As dietas, cuja composição químico-bromatológica se encontra na Tabela 1, apresentaram níveis crescentes de concentrado de 45, 60, 75 e 90\%.

As rações foram fornecidas, em uma refeição diária, às $7 \mathrm{~h} 30$, à vontade, ajustando-se sobra diária em torno de $10 \%$ do oferecido, por animal, e registrandose a ingestão durante todo o período experimental.

Ao atingirem os pesos preestabelecidos de $190 \pm 10 \mathrm{~kg}$ (grupo 1) e $300 \pm 10 \mathrm{~kg}$ (grupo 2), os animais foram abatidos. Antes de serem abatidos, os animais foram submetidos a período de jejum por 16 horas, com acesso à água. $\mathrm{O}$ abate foi feito por concussão cerebral e secção da veia jugular e, após o sacrifício, retiraram-se o sangue, o couro, a cabeça, os pés, o mesentério, a gordura interna, o TGI cheio e a MOI (fígado, coração, pulmão, rins e baço) e procedeu-se à pesagem. Em seguida, o TGI vazio e a MOI foram seccionados, separando-se as partes constituintes, que foram lavadas em água corrente e, após escorrer o excesso de água, foram pesadas e amostradas. Os comprimentos dos intestinos foram mensurados com fita métrica.

O peso corporal vazio (PCVZ) foi obtido pela soma dos pesos da carcaça, do sangue, da cabeça, do couro, dos pés, da cauda, das vísceras e dos órgãos.

Utilizou-se o delineamento experimental inteiramente casualizado, com quatro tratamentos e quatro repetições para ambos os grupos. Realizaram-se análises de variância e regressão na interpretação das variáveis estudadas, usando-se o programa computacional SAEG (Sistema de Análises Estatísticas e Genéticas), desenvolvido por EUCLYDES (1983). Os coeficientes de regressão foram comparados pelo teste "t", adotando-se os níveis de 5 e $10 \%$ de probabilidade.

\section{Resultados e Discussão}

Não houve diferenças com relação aos pesos médios, da maioria dos componentes do TGI e comprimentos dos ID e IG, expressos em valores absolutos, quando os animais do grupo 1 e 2 receberam as dietas contendo quatro níveis de concentrado (Tabela 2). Os resultados do presente estudo foram semelhantes aos encontrados por ARAÚJO et al. (1996), que trabalharam com animais mestiços Holandês x Zebu abatidos aos $300 \mathrm{~kg}$ de peso vivo (PV) e submetidos a sistema de alimentação similar.

O peso do omaso, para os animais do grupo 1 , apresentou comportamento quadrático $(\mathrm{P}<0,05)$, estimando-se o peso mínimo de 1,23 kg para o nível de
Tabela 1 - Teores de matéria seca (MS), matéria orgânica (MO), proteína bruta (PB), extrato etéreo (EE), nutrientes digestíveis totais (NDT), carboidratos totais (CHOT), fibra em detergente neutro (FDN), $\mathrm{Ca}, \mathrm{P}, \mathrm{Mg}, \mathrm{K}$ e Na

Table 1 - Contents of dry matter (DM), organic matter (OM), crude protein (CP), ether extract (EE), total digestible nutrients $(T D N)$, total carbohydrates (TCH), neutral detergent fiber (NDF), Ca, $\mathrm{P}, \mathrm{Mg}, \mathrm{K}$ and $\mathrm{Na}$

\begin{tabular}{lcccc}
\hline \multirow{2}{*}{ Item } & \multicolumn{4}{c}{$\begin{array}{c}\text { Nível de concentrado (\%) } \\
\text { Level of concentrate }\end{array}$} \\
\cline { 2 - 5 } & 45 & 60 & 75 & 90 \\
\hline MS , \% & 87,51 & 87,39 & 87,45 & 87,43
\end{tabular}

$D M$

$\begin{array}{lllll}\mathrm{MO}^{1} & 95,46 & 95,81 & 96,23 & 96,41 \\ O M & & & \end{array}$

$\begin{array}{lllll}\mathrm{PB}^{1} & 15,68 & 15,48 & 15,79 & 15,68\end{array}$

$\begin{array}{lrrrr}C P & & & & \\ \mathrm{EE}^{1} & 1,90 & 2,71 & 2,89 & 3,01\end{array}$

$\begin{array}{lrrr}\text { NDT }^{1} & 69,67 & 73,33 & 76,33\end{array}$

TDN

$\begin{array}{lllll}\text { CHOT }^{1} & 77,33 & 77,10 & 77,05 & 77,20\end{array}$

$\begin{array}{lllll}T C H & & & & \\ \text { FDN }^{1} & 52,91 & 41,94 & 32,88 & 24,27\end{array}$

$\begin{array}{lllll}N D F & & & & \\ \mathrm{Ca}^{1} & 0,55 & 0,52 & 0,51 & 0,54\end{array}$

$\begin{array}{lllll}\mathrm{P}^{1} & 0,22 & 0,25 & 0,20 & 0,20\end{array}$

$\begin{array}{lllll}\mathrm{Mg}^{1} & 0,13 & 0,13 & 0,12 & 0,11\end{array}$

$\mathrm{K}^{1} \quad 1,45 \quad 1,28 \quad 1,06 \quad 0,92$

\begin{tabular}{lllll}
$\mathrm{Na}^{1}$ & 0,10 & 0,13 & 0,12 & 0,16 \\
\hline
\end{tabular}

$1 \% \mathrm{MS}(\% D M)$.

$72,68 \%$ de concentrado na dieta. Johnson et al. 1985, citados por DROUILLARD et al. (1991), relataram que bovinos alimentados com dietas contendo proporções elevadas de feno de alfafa apresentaram maiores pesos do omaso. Da mesma forma, JONES et al. (1985) encontraram maiores pesos de omaso para animais alimentados com dietas à base de volumoso ( $50 \%$ de silagem de milho $+50 \%$ de feno), quando comparados com animais que receberam dietas à base de concentrado ( $30 \%$ de silagem de milho $+70 \%$ de milho moído, com base na matéria seca).

O peso do abomaso, em valores absolutos, para os animais do grupo 1 e 2 , apresentou comportamento linear crescente, com a inclusão de concentrado na dieta.

Os pesos médios da MOI, expressos em valores absolutos, para os animais do grupo 2, não foram influenciados pela adição de concentrado nas dietas (Tabela 3 ).

Os pesos do coração e do baço, em valores absolutos, para os animais do grupo 1, apresentaram comportamento quadrático, estimando-se os pesos mínimos de 0,78 e $0,53 \mathrm{~kg}$, para os níveis de 57,56 e $65,74 \%$ de concentrado na dieta, respectivamente. 
SIGNORETTI et al.

Tabela 2 - Médias e regressão ajustada, para os pesos $(\mathrm{kg})$ dos constituintes do trato gastrintestinal (TGI) e comprimentos (m) dos intestinos delgado (CID) e grosso (CIG), em valores absolutos, para animais dos grupos 1 (190 kg PV) e do grupo 2 (300 kg PV)

Table 2 - Means and fitted regression for weight of the gastrintrestinal tract (GIT) and lengths (m) of small intestine (LSI) and large (LLI), in absolute values, for animals of group 1 (190 kg LW) and of group 2 (300 kg LW)

\begin{tabular}{|c|c|c|c|c|c|c|}
\hline \multirow[t]{2}{*}{ Item } & \multicolumn{4}{|c|}{$\begin{array}{c}\text { Nível de concentrado (\%) } \\
\text { Level of concentrate }\end{array}$} & \multirow[t]{2}{*}{$\mathrm{CV}(\%)$} & \multirow[t]{2}{*}{$\begin{array}{c}\text { Regressão } \\
\text { Regression }\end{array}$} \\
\hline & 45 & 60 & 75 & 90 & & \\
\hline \multicolumn{7}{|c|}{ Grupo 1 (Group 1) ${ }^{1}$} \\
\hline Retículo-rúmen(Reticulum-rumen) & 3,35 & 3,91 & 3,42 & 4,01 & 12,97 & $\hat{\mathrm{Y}}=3,67$ \\
\hline Omaso (Omasum) & 1,44 & 1,28 & 1,23 & 1,31 & 16,73 & 1 \\
\hline Abomaso (Abomasum) & 0,65 & 0,72 & 0,71 & 0,76 & 10,71 & 2 \\
\hline Peso ID (Weight SI) & 4,68 & 5,11 & 4,27 & 4,05 & 24,15 & $\hat{\mathrm{Y}}=4,53$ \\
\hline Peso IG (Weight LI) & 2,04 & 2,18 & 1,96 & 2,08 & 11,25 & $\hat{\mathrm{Y}}=2,06$ \\
\hline $\mathrm{CID}(L S I)$ & 36,20 & 37,64 & 37,76 & 35,89 & 8,75 & $\hat{\mathrm{Y}}=36,87$ \\
\hline $\mathrm{CIG}(L L I)$ & 7,61 & 8,17 & 7,21 & 7,58 & 9,13 & $\hat{\mathrm{Y}}=7,64$ \\
\hline Total TGI (Total GIT), kg & 12,15 & 13,19 & 11,60 & 12,21 & 13,19 & $\hat{\mathrm{Y}}=12,29$ \\
\hline \multicolumn{7}{|c|}{ Grupo 2 (Group 2) ${ }^{2}$} \\
\hline Retículo-rúmen (Reticulum-rumen) & 4,94 & 4,88 & 5,30 & 4,94 & 6,60 & $\hat{\mathrm{Y}}=5,01$ \\
\hline Omaso (Omasum) & 2,16 & 1,59 & 2,21 & 1,61 & 20,60 & $\hat{\mathrm{Y}}=1,89$ \\
\hline Abomaso (Abomasum) & 0,89 & 0,94 & 0,97 & 1,03 & 8,88 & 3 \\
\hline Peso ID (Weight SI) & 6,02 & 6,15 & 6,19 & 6,38 & 10,51 & $\hat{\mathrm{Y}}=6,18$ \\
\hline Peso IG (Weight LI) & 2,83 & 2,96 & 2,61 & 3,00 & 12,69 & $\hat{\mathrm{Y}}=2,06$ \\
\hline $\mathrm{CID}(L S I)$ & 40,90 & 43,20 & 42,18 & 41,58 & 5,35 & $\hat{\mathrm{Y}}=41,96$ \\
\hline $\mathrm{CIG}(L L I)$ & 8,80 & 9,30 & 8,60 & 8,41 & 9,39 & $\hat{\mathrm{Y}}=8,78$ \\
\hline Total TGI (Total GIT), kg & 16,85 & 16,52 & 17,28 & 16,96 & 6,29 & $\hat{\mathrm{Y}}=16,90$ \\
\hline \multicolumn{5}{|c|}{ 1. $\hat{\mathrm{Y}}=2,65287-0,039158^{*} \mathrm{NC}+0,0002694^{*} \mathrm{NC}^{2}$} & & $\mathrm{R}^{2}=0,99$ \\
\hline \multicolumn{5}{|l|}{ 2. $\hat{\mathrm{Y}}=0,5625+0,002166{ }^{* * *} \mathrm{NC}$} & & $r^{2}=0,85$ \\
\hline \multicolumn{7}{|c|}{$\begin{array}{lr}\text { 3. } \hat{\mathrm{Y}}=0,7575+0,0030 * \mathrm{NC} & \mathrm{r}^{2}=0,99\end{array}$} \\
\hline
\end{tabular}

Tabela 3 - Médias e regressão ajustada, para os pesos $(\mathrm{kg})$ da massa de órgãos internos (MOI), em valores absolutos, para animais dos grupos 1 (190 kg PV) e 2 (300 kg PV)

Table 3 - Means and fitted regression for weight of the mass of internal organs (MIO), in absolute values, for animals of group 1 (190 $\mathrm{kg} \mathrm{LW})$ and $2(300 \mathrm{~kg} \mathrm{LW})$

\begin{tabular}{|c|c|c|c|c|c|c|}
\hline \multirow[t]{2}{*}{ Item } & \multicolumn{3}{|c|}{$\begin{array}{c}\text { Nível de concentrado (\%) } \\
\text { Level of concentrate }\end{array}$} & & \multirow[t]{2}{*}{$\mathrm{CV}(\%)$} & \multirow[t]{2}{*}{$\begin{array}{c}\text { Regressão } \\
\text { Regression }\end{array}$} \\
\hline & 45 & 60 & 75 & 90 & & \\
\hline \multicolumn{7}{|c|}{ Grupo 1 (Group 1) 1} \\
\hline Fígado (Liver) & 3,32 & 3,34 & 3,55 & 3,87 & 9,84 & 1 \\
\hline Pulmão(Lung) & 1,83 & 1,80 & 1,84 & 2,18 & 13,48 & 2 \\
\hline Coração (Heart) & 0,84 & 0,73 & 0,92 & 1,08 & 11,31 & 3 \\
\hline Rins (Kidneys) & 0,61 & 0,62 & 0,69 & 0,83 & 13,59 & 4 \\
\hline Baço (Spleen) & 0,70 & 0,52 & 0,59 & 0,75 & 24,81 & 5 \\
\hline Total MOI (Total MIO), kg & 7,30 & 7,01 & 7,59 & 8,71 & 8,83 & 6 \\
\hline \multicolumn{7}{|c|}{ Grupo 2 (Group 2) ${ }^{2}$} \\
\hline Figado (Liver) & 4,64 & 5,01 & 4,93 & 5,21 & 11,30 & $\hat{\mathrm{Y}}=4,95$ \\
\hline Pulmão (Lung) & 2,68 & 2,47 & 2,61 & 2,75 & 13,06 & $\hat{\mathrm{Y}}=2,63$ \\
\hline Coração (Heart) & 1,30 & 1,28 & 1,33 & 1,45 & 13,51 & $\hat{\mathrm{Y}}=1,34$ \\
\hline Rins (Kidneys) & 1,01 & 0,82 & 0,92 & 0,94 & 15,72 & $\hat{\mathrm{Y}}=0,92$ \\
\hline Baço (Spleen) & 1,01 & 0,82 & 0,86 & 1,09 & 38,00 & $\hat{\mathrm{Y}}=0,95$ \\
\hline Total MOI (Total MIO), kg & 10,64 & 10,40 & 10,65 & 11,43 & 10,61 & $\hat{\mathrm{Y}}=10,78$ \\
\hline \multicolumn{6}{|l|}{ 1. $\hat{\mathrm{Y}}=2,6885+0,01230^{*} \mathrm{NC}$} & $r^{2}=0,88$ \\
\hline \multicolumn{6}{|l|}{ 2. $\hat{\mathrm{Y}}=1,4332+0,00710^{\star * *} \mathrm{NC}$} & $r^{2}=0,61$ \\
\hline \multicolumn{6}{|c|}{ 3. $\hat{\mathrm{Y}}=1,7687-0,034499^{*} \mathrm{NC}+0,0002999^{*} \mathrm{NC}^{2}$} & $\mathrm{R}^{2}=0,92$ \\
\hline \multicolumn{6}{|l|}{ 4. $\hat{\mathrm{Y}}=0,3525+0,004916^{\star} \mathrm{NC}$} & $r^{2}=0,88$ \\
\hline \multicolumn{6}{|c|}{ 5. $\hat{\mathrm{Y}}=2,1523-0,0493082^{\star * *} \mathrm{NC}+0,0003749^{\star * *} \mathrm{NC}^{2}$} & $\mathrm{R}^{2}=0,97$ \\
\hline \multicolumn{7}{|c|}{ 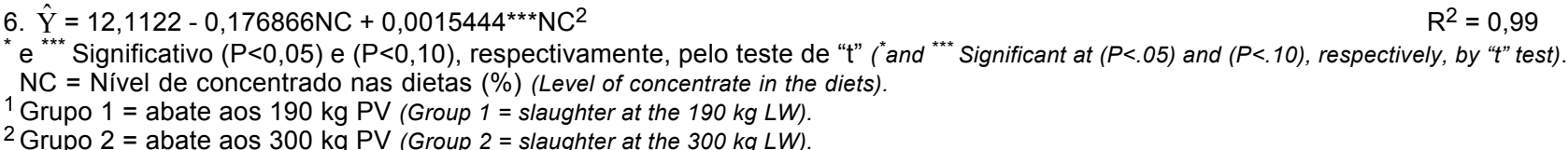 } \\
\hline
\end{tabular}


Contudo, o peso do fígado, do pulmão e dos rins apresentaram comportamento linear crescente, com a inclusão de concentrado nas dietas. ARAÚJO et al. (1996) verificaram comportamento quadrático para o peso do fígado, em animais mestiços Holandês x Zebu abatidos com $300 \mathrm{~kg}$ de peso.

O peso total da MOI apresentou comportamento quadrático, estimando-se o peso mínimo de $7,05 \mathrm{~kg}$ para o nível de 57,26\% de concentrado na dieta (Tabela3). De maneira geral, os animais abatidos com 190 ou 300 $\mathrm{kg}$ de PV, alimentados com dietas contendo maiores níveis de concentrado, que, conseqüentemente, consumiram mais energia em suas dietas, apresentaram maior tamanho relativo dos órgãos vitais.

LUNT et al. (1986) observaram que novilhos alimentados com dietas à base de volumosos tiveram maiores pesos de coração e fígado que os alimentados com dietas à base de grãos. Por outro lado, o fígado de ruminantes em crescimento respondeu linearmente ao aumento no consumo de energia metabolizável (JOHNSON et al., 1990).

Nas Tabelas 4 e 5, são mostrados os pesos médios das partes do TGI e MOI, respectivamente, expressos por $100 \mathrm{~kg}$ de PCVZ, para os animais dos grupos 1 e 2 . Os pesos do TGI e os comprimentos do ID e IG dos animais abatidos com 190 e $300 \mathrm{~kg}$ de PV não foram influenciados pelo nível de concentrado nas dietas. Entretanto, o comprimento do ID dos animais abatidos com $300 \mathrm{~kg}$ de PV diminuiu linearmente $(\mathrm{P}<0,05)$ com aumento do nível de concentrado na dieta.

ARAÚJO et al. (1996), trabalhando com bezerros mestiços Holandês x Zebu submetidos a sistema de alimentação similar e abatidos aos $300 \mathrm{~kg}$ de PV, observaram redução linear no peso do omaso e do ID com adição de concentrado nas dietas.

Johnson et al. (1985), citados por DROUILLARD et al. (1991), relataram que bovinos alimentados com dietas contendo proporções elevadas de feno de alfafa apresentaram pouco ou nenhum efeito nos pesos do rúmen, ceco e cólon. No entanto, os pesos dos compartimentos do TGI, expressos em porcentagem do PCVZ, apresentaram maior desenvolvimento na fase mais precoce da vida do animal, desacelerando-se mais cedo que os tecidos muscular e adiposo (BERG e BUTTERFIELD, 1976).

O peso da MOI, expresso por $100 \mathrm{~kg}$ de PCVZ,

Tabela 4 - Médias e regressão ajustadas para os pesos $(\mathrm{kg})$ dos constituintes do trato gastrintestinal (TGI) e comprimentos (m) dos intestinos delgado (CID) e grosso (CIG), por $100 \mathrm{~kg} \mathrm{PVC,} \mathrm{para} \mathrm{animais} \mathrm{do} \mathrm{grupo} 1$ (190 kg PV) e 2 (300 kg PV)

Table 4 - Means and fitted regression for weight of the gastrintestinal tract (GIT) and lengths (m) of small intestine (LSI) and large (LLI), per $100 \mathrm{~kg}$ EBW, for animals of group 1 (190 kg LW) and 2 (300 kg LW)

\begin{tabular}{|c|c|c|c|c|c|c|}
\hline \multirow[t]{2}{*}{$\overline{\text { Item }}$} & \multicolumn{4}{|c|}{$\begin{array}{l}\text { Nível de concentrado }(\%) \\
\text { Level of concentrate }\end{array}$} & \multirow[t]{2}{*}{$\mathrm{CV}(\%)$} & \multirow[t]{2}{*}{$\begin{array}{l}\text { Regressão } \\
\text { Regression }\end{array}$} \\
\hline & 45 & 60 & 75 & 90 & & \\
\hline \multicolumn{7}{|c|}{ Grupo $1(\text { Group } 1)^{1}$} \\
\hline Retículo-rúmen (Reticulum-rumen) & 2,11 & 2,35 & 2,10 & 2,36 & 10,90 & $\hat{\mathrm{Y}}=2,23$ \\
\hline Omaso (Omasum) & 0,91 & 0,77 & 0,75 & 0,77 & 14,50 & $\hat{\mathrm{Y}}=0,80$ \\
\hline Abomaso (Abomasum) & 0,41 & 0,43 & 0,48 & 0,45 & 10,48 & $\hat{\mathrm{Y}}=0,44$ \\
\hline Peso ID (Weight SI) & 2,95 & 3,08 & 2,62 & 2,38 & 22,58 & $\hat{Y}=2,76$ \\
\hline Peso IG (Weight LI) & 1,28 & 1,31 & 1,21 & 1,23 & 11,88 & $\hat{\mathrm{Y}}=1,26$ \\
\hline $\mathrm{CID}(L S I)$ & 22,88 & 22,66 & 23,32 & 21,10 & 9,84 & $\hat{\mathrm{Y}}=22,49$ \\
\hline $\mathrm{CIG}(L L I)$ & 4,78 & 4,92 & 4,46 & 4,47 & 10,12 & $\hat{\mathrm{Y}}=4,66$ \\
\hline Total GIT (Total GIT), $\mathrm{kg}$ & 7,66 & 7,94 & 7,11 & 7,19 & 11,43 & $\hat{\mathrm{Y}}=7,47$ \\
\hline \multicolumn{7}{|c|}{ Grupo 2 (Group 2$)^{2}$} \\
\hline Retículo-rúmen(Reticulum-rumen) & 1,93 & 1,82 & 1,98 & 1,84 & 7,50 & $\hat{\mathrm{Y}}=1,89$ \\
\hline Omaso (Omasum) & 0,85 & 0,59 & 0,83 & 0,60 & 20,47 & $\hat{\mathrm{Y}}=0,71$ \\
\hline Abomaso (Abomasum) & 0,35 & 0,35 & 0,36 & 0,38 & 8,50 & $\hat{\mathrm{Y}}=0,36$ \\
\hline Peso ID (Weight SI) & 2,35 & 2,29 & 2,31 & 2,37 & 11,07 & $\hat{\mathrm{Y}}=2,33$ \\
\hline Peso IG (Weight LI) & 1,11 & 1,11 & 0,98 & 1,12 & 13,45 & $\hat{\mathrm{Y}}=1,08$ \\
\hline $\mathrm{CID}(L S I)$ & 16,01 & 16,07 & 15,74 & 15,42 & 6,29 & 1 \\
\hline $\mathrm{CIG}(L L I)$ & 3,45 & 3,46 & 3,22 & 3,12 & 10,46 & $\hat{\mathrm{Y}}=3,31$ \\
\hline Total GIT (Total GTI), kg & 6,59 & 6,15 & 6,45 & 6,30 & 7,13 & $\hat{\mathrm{Y}}=6,37$ \\
\hline 1. $\hat{\mathrm{Y}}=16,7465-0,0138834^{*} \mathrm{NC}$ & & & & & & $\mathrm{r}^{2}=0,83$ \\
\hline
\end{tabular}


Tabela 5 - Média e regressão ajustada dos pesos ( $\mathrm{kg}$ ) da massa de órgãos internos (MOI), por $100 \mathrm{~kg}$ PCV, para animais dos grupos 1 (190 kg PV) e 2 (300 kg PV)

Table 5 - Mean and fitted regression of the weights of the mass of internal organs (MIO), per $100 \mathrm{~kg}$ EBW, for animals of groups 1 (190 $\mathrm{kg} L W)$ and $2(300 \mathrm{~kg} L W)$

\begin{tabular}{|c|c|c|c|c|c|c|}
\hline \multirow[t]{2}{*}{ Item } & \multicolumn{4}{|c|}{$\begin{array}{l}\text { Nível de concentrado (\%) } \\
\text { Level of concentrate }\end{array}$} & \multirow[t]{2}{*}{ CV (\%) } & \multirow[t]{2}{*}{$\begin{array}{l}\text { Regressão } \\
\text { Regression }\end{array}$} \\
\hline & 45 & 60 & 75 & 90 & & \\
\hline \multicolumn{7}{|c|}{ Grupo 1 (Group 1) 1} \\
\hline Fígado (Liver) & 2,09 & 2,01 & 2,18 & 2,27 & 8,10 & 1 \\
\hline Pulmão (Lung) & 1,16 & 1,09 & 1,14 & 1,28 & 12,58 & $\hat{\mathrm{Y}}=1,16$ \\
\hline Coração (Heart) & 0,53 & 0,44 & 0,56 & 0,64 & 10,01 & 2 \\
\hline Rins (Kidneys) & 0,38 & 0,37 & 0,43 & 0,48 & 14,03 & 3 \\
\hline Baço (Spleen) & 0,44 & 0,31 & 0,36 & 0,44 & 25,95 & 4 \\
\hline Total MOI (Total MIO), kg & 4,60 & 4,22 & 4,67 & 5,11 & 7,80 & 5 \\
\hline \multicolumn{7}{|c|}{ Grupo 2 (Group 2) ${ }^{2}$} \\
\hline Figado (Liver) & 1,81 & 1,86 & 1,84 & 1,93 & 10,52 & $\hat{\mathrm{Y}}=1,86$ \\
\hline Pulmão (Lung) & 1,04 & 0,92 & 0,97 & 1,02 & 12,27 & $\hat{\mathrm{Y}}=0,99$ \\
\hline Coração (Heart) & 0,51 & 0,48 & 0,50 & 0,54 & 13,04 & $\hat{\mathrm{Y}}=0,50$ \\
\hline Rins (Kidneys) & 0,40 & 0,31 & 0,34 & 0,35 & 16,53 & $\hat{\mathrm{Y}}=0,35$ \\
\hline Baço (Spleen) & 0,40 & 0,31 & 0,33 & 0,40 & 37,23 & $\hat{\mathrm{Y}}=0,36$ \\
\hline Total MOI (Total MIO), kg & 4,16 & 3,86 & 3,97 & 4,24 & 9,84 & $\hat{\mathrm{Y}}=4,06$ \\
\hline \multicolumn{5}{|l|}{ 1. $\hat{\mathrm{Y}}=1,8237+0,004666^{* * *} \mathrm{NC}$} & & $r^{2}=0,66$ \\
\hline \multicolumn{6}{|c|}{ 2. $\hat{Y}=1,1010-0,021033^{\star} N C+0,0001777^{*} N C^{2}$} & $R^{2}=0,83$ \\
\hline \multicolumn{6}{|c|}{ 3. $\hat{\mathrm{Y}}=0,2525+0,002416^{*} \mathrm{NC}$} & $r^{2}=0,88$ \\
\hline \multicolumn{6}{|c|}{ 4. $\hat{\mathrm{Y}}=1,3400-0,030416^{\star * *} \mathrm{NC}+0,0002277^{* * *} \mathrm{~N} \mathrm{C}^{2}$} & $R^{2}=0,90$ \\
\hline \multicolumn{6}{|c|}{ 5. $\hat{\mathrm{Y}}=7,6506-0,109541^{* * *} \mathrm{NC}+0,0009083^{* * *} N C^{2}$} & $\mathrm{R}^{2}=0,91$ \\
\hline
\end{tabular}

não foi influenciado pelo nível de concentrado nas dietas, para os animais do grupo 2 (Tabela 5), entretanto, os pesos do fígado e dos rins dos animais do grupo 1 apresentaram comportamento linear crescente, com adição de concentrado nas dietas. Observou-se que os animais alimentados com dietas contendo $90 \%$ de concentrado tiveram os maiores pesos do fígado e dos rins.

Os pesos do coração, do baço e do total da MOI, em \% PCVZ, para os animais do grupo 1, apresentaram comportamento quadrático, estimando-se os pesos mínimos de 0,$48 ; 0,32$; e 4,35 kg para os níveis de 59,$16 ; 66,77$; e $60,30 \%$ de concentrado na dieta, respectivamente (Tabela 5 ).

Em animais de raças leiteiras submetidos ao consumo à vontade, os órgãos internos representaram menor proporção de PCVZ em animais mais jovens e o peso do fígado, em termos proporcionais, reduziu com relação ao PCVZ, em animais submetidos a baixo nível nutricional (PERON et al., 1993).

Os pesos da cabeça, dos pés, do couro e da gordura interna, expressos em valores absolutos e \% PCVZ, para os animais do grupo 1, não foram influenciados pelo nível de concentrado nas dietas (Tabelas 6 e 7). O peso do sangue (em valores absolutos e em \% PCVZ) e o peso do total destas partes não-integrantes da carcaça (em valores absolutos), para os animais do grupo 1, aumentaram linearmente $(\mathrm{P}<0,05)$, enquanto o peso do sangue (em \% PCVZ) e o peso total destas partes não-integrantes da carcaça (em valores absolutos), para os animais do grupo 2, apresentaram comportamento quadrático, estimando-se os pesos máximos de 5,16e $75,42 \mathrm{~kg}$ para os níveis de 66,29 e $75,79 \%$ de concentrado na dieta, respectivamente.

$\mathrm{O}$ peso do mesentério (em valores absolutos e \% PCVZ), para os animais abatidos com $190 \mathrm{~kg}$ de $\mathrm{PV}$, apresentou comportamento quadrático, estimando-se os pesos mínimos de 3,46 e 2,13 kg para os níveis de 58,75 e $59,98 \%$ de concentrado na dieta, respectivamente.

Os animais do grupo 2 apresentaram aumento $(\mathrm{P}<0,10)$ no peso do mesentério e reduçãolinear $(\mathrm{P}<0,05)$ no peso da cabeça (em peso absoluto e \% PCVZ), enquanto os pesos do couro, da gordura interna e do total destes componentes não foram influenciados pelo aumento da proporção de concentrado na dieta.

O peso dos pés, em \% PCVZ, para os animais do grupo 2, apresentou comportamento quadrático, estimando-se o peso mínimo de $2,88 \mathrm{~kg}$ para o nível de 
Rev. bras. zootec.

Tabela 6 - Médias e regressão ajustada, para os pesos (kg) de cabeça, pés, couro, mesentério (MESEN), gordura interna (GI), sangue e do total, em valores absolutos, para animais dos grupos 1 (190 kg PV) e 2 (300 kg PV)

Table 6 - Means and fitted regression for weight of the head, foot, hide, mesenteric (MESEN), internal fat (IF), blood and the total, in absolute values, for animals of groups 1 (190 kg LW) and $2(300 \mathrm{~kg} \mathrm{LW})$

\begin{tabular}{|c|c|c|c|c|c|c|}
\hline \multirow[t]{2}{*}{ Item } & \multicolumn{4}{|c|}{$\begin{array}{l}\text { Nível de concentrado (\%) } \\
\text { Level of concentrate }\end{array}$} & \multirow[t]{2}{*}{$\mathrm{CV}(\%)$} & \multirow[t]{2}{*}{$\begin{array}{l}\text { Regressão } \\
\text { Regression }\end{array}$} \\
\hline & 45 & 60 & 75 & 90 & & \\
\hline \multicolumn{7}{|c|}{ Grupo 1 (Group 1) ${ }^{1}$} \\
\hline Cabeça (Head) & 7,90 & 7,95 & 7,45 & 8,13 & 5,25 & $\hat{\mathrm{Y}}=7,86$ \\
\hline Pés (Foot) & 5,28 & 5,58 & 5,33 & 5,28 & 5,67 & $\hat{\mathrm{Y}}=5,36$ \\
\hline Couro (Hide) & 14,45 & 14,83 & 14,53 & 15,01 & 6,22 & $\hat{\mathrm{Y}}=14,70$ \\
\hline MESEN & 3,69 & 3,74 & 3,63 & 5,23 & 16,00 & 1 \\
\hline $\mathrm{GI}(I F)$ & 1,97 & 2,48 & 2,57 & 2,33 & 29,97 & $\hat{\mathrm{Y}}=2,34$ \\
\hline Sangue (Blood) & 8,68 & 9,27 & 9,86 & 10,07 & 7,86 & 2 \\
\hline Total & 41,97 & 43,83 & 43,37 & 46,03 & 4,13 & $\hat{\mathrm{Y}}=43,80$ \\
\hline \multicolumn{7}{|c|}{ Grupo $2(\text { Group } 2)^{2}$} \\
\hline Cabeça (Head) & 13,13 & 13,08 & 12,85 & 12,10 & 6,22 & 3 \\
\hline Pés (Foot) & 8,30 & 7,93 & 7,83 & 8,30 & 6,60 & $\hat{\mathrm{Y}}=8,09$ \\
\hline Couro (Hide) & 26,30 & 27,38 & 25,93 & 26,65 & 5,95 & $\hat{\mathrm{Y}}=26,55$ \\
\hline MESEN & 6,16 & 8,46 & 8,87 & 10,03 & 18,22 & 4 \\
\hline $\mathrm{GI}(I F)$ & 3,89 & 4,55 & 5,24 & 4,89 & 22,08 & $\hat{\mathrm{Y}}=4,64$ \\
\hline Sangue (Blood) & 12,48 & 13,21 & 14,28 & 12,53 & 6,61 & 5 \\
\hline Total & 70,25 & 74,54 & 74,99 & 74,50 & 4,01 & 6 \\
\hline \multicolumn{5}{|c|}{ 1. $\hat{\mathrm{Y}}=9,3814-0,201716^{* * *} \mathrm{NC}+0,0017166^{* * *} \mathrm{NC}^{2}$} & & $R^{2}=0,90$ \\
\hline \multicolumn{5}{|c|}{ 2. $\hat{\mathrm{Y}}=7,32725+0,031716^{*} \mathrm{NC}$} & & $r^{2}=0,96$ \\
\hline \multicolumn{5}{|c|}{ 3. $\hat{\mathrm{Y}}=14,2725-0,02200^{* * *} \mathrm{NC}$} & & $r^{2}=0,92$ \\
\hline \multicolumn{5}{|c|}{ 4. $\hat{\mathrm{Y}}=2,96525+0,08020 * \mathrm{NC}$} & & $r^{2}=0,81$ \\
\hline \multicolumn{5}{|c|}{ 5. $\hat{\mathrm{Y}}=0,8001+0,379807^{\star} \mathrm{NC}-0,0027527^{\star} \mathrm{N} \mathrm{C}^{2}$} & & $R^{2}=0,76$ \\
\hline \multicolumn{5}{|c|}{ 6. $\hat{\mathrm{Y}}=44,9485+0,804215^{\star * \star} \mathrm{NC}-0,0053055^{\star * \star} \mathrm{NC}^{2}$} & & $\mathrm{R}^{2}=0,97$ \\
\hline
\end{tabular}

Tabela 7 - Médias e regressão ajustadas, para os pesos $(\mathrm{kg})$ de cabeça, pés, couro, mesentério (MESEN), gordura interna (GI), sangue e do total, por $100 \mathrm{~kg} \mathrm{PCV}$, para animais dos grupos 1 (190 kg PV) e 2 (300 kg PV)

Table 7 - Means and fitted regression for weight of the head, foot, hide, mesenteric (MESEN), internal fat (IF), blood and the total, per $100 \mathrm{~kg}$ EBW, for animals of groups $1(190 \mathrm{~kg} \mathrm{LW})$ and $2(300 \mathrm{~kg} \mathrm{LW})$

\begin{tabular}{|c|c|c|c|c|c|c|}
\hline \multirow{3}{*}{$\begin{array}{l}\text { Parte } \\
\text { Part }\end{array}$} & \multirow{2}{*}{\multicolumn{4}{|c|}{$\begin{array}{l}\text { Nível de concentrado (\%) } \\
\text { Level of concentrate }\end{array}$}} & \multirow[t]{3}{*}{ CV (\%) } & \multirow{3}{*}{$\begin{array}{l}\text { Regressão } \\
\text { Regression }\end{array}$} \\
\hline & & & & & & \\
\hline & 45 & 60 & 75 & 90 & & \\
\hline \multicolumn{7}{|c|}{ Grupo 1 (Group 1) 1} \\
\hline Cabeça (Head) & 4,73 & 4,79 & 4,59 & 4,79 & 6,52 & $\hat{\mathrm{Y}}=4,72$ \\
\hline Pés (Foot) & 3,33 & 3,36 & 3,29 & 3,10 & 5,76 & $\hat{\mathrm{Y}}=3,27$ \\
\hline Couro (Hide) & 9,12 & 8,93 & 8,93 & 8,85 & 6,23 & $\hat{\mathrm{Y}}=8,96$ \\
\hline MESEN & 2,32 & 2,25 & 2,24 & 3,08 & 15,51 & 1 \\
\hline $\mathrm{GI}(I F)$ & 1,24 & 1,49 & 1,53 & 1,37 & 28,58 & $\hat{Y}=1,42$ \\
\hline Sangue (Blood) & 5,47 & 5,58 & 6,08 & 5,93 & 7,53 & 2 \\
\hline Total & 26,46 & 26,40 & 26,70 & 27,11 & 3,69 & $\hat{\mathrm{Y}}=26,67$ \\
\hline \multicolumn{7}{|c|}{ Grupo 2 (Group 2) ${ }^{2}$} \\
\hline Cabeça (Head) & 5,12 & 4,86 & 4,80 & 4,50 & 6,54 & 3 \\
\hline Pés (Foot) & 3,25 & 2,95 & 2,92 & 3,08 & 7,07 & 4 \\
\hline Couro (Hide) & 10,28 & 10,16 & 9,67 & 9,89 & 6,29 & $\hat{\mathrm{Y}}=10,00$ \\
\hline MESEN & 2,40 & 3,15 & 3,31 & 3,71 & 16,76 & 5 \\
\hline $\mathrm{GI}(I F)$ & 1,52 & 1,69 & 1,95 & 1,81 & 21,84 & $\hat{\mathrm{Y}}=1,74$ \\
\hline Sangue (Blood) & 4,88 & 4,92 & 5,32 & 4,65 & 7,55 & 6 \\
\hline Total & 27,46 & 27,73 & 27,97 & 27,64 & 3,90 & $\hat{\mathrm{Y}}=27,70$ \\
\hline \multicolumn{5}{|c|}{ 1. $\hat{\mathrm{Y}}=5,7484-0,120616^{\star} \mathrm{NC}+0,001005^{\star} \mathrm{NC}^{2}$} & & $R^{2}=0,94$ \\
\hline \multicolumn{5}{|c|}{ 2. $\hat{\mathrm{Y}}=4,9290+0,0120616^{\star \star *} \mathrm{NC}$} & & $r^{2}=0,71$ \\
\hline \multicolumn{5}{|c|}{ 3. $\hat{\mathrm{Y}}=5,7042-0,013100^{*} \mathrm{NC}$} & & $r^{2}=0,95$ \\
\hline \multicolumn{5}{|c|}{ 4. $\hat{\mathrm{Y}}=5,45312-0,072041^{* \star *} \mathrm{NC}+0,0005083^{* \star *} \mathrm{NC}^{2}$} & & $R^{2}=0,99$ \\
\hline \multirow{2}{*}{\multicolumn{5}{|c|}{ 5. $\hat{\mathrm{Y}}=1,30475+0,027216^{*} \mathrm{NC}$}} & & $r^{2}=0,93$ \\
\hline & \multicolumn{4}{|c|}{ 6. $\hat{\mathrm{Y}}=1,7333+0,103491^{\star \star *} \mathrm{NC}-0,0007805^{\star \star \star} \mathrm{NC} \mathrm{C}^{2}$} & & $\mathrm{R}^{2}=0,55$ \\
\hline
\end{tabular}


$70,86 \%$ de concentrado na dieta.

Observou-se que os animais do grupo 2, alimentados com dietas contendo $90 \%$ de concentrado, apresentaram maior quantidade de gordura mesentérica (em valores absolutos e em \% PCVZ) em relação aos que receberam dietas contendo $45 \%$ de concentrado (Tabelas 6 e 7). Estes resultados estão de acordo com os obtidos por Kauffman et al. (1976), citados por JONES et al. (1985), que verificaram maiores quantidades de gordura visceral em animais alimentados com dietas altamente energéticas em relação aos que receberam dietas moderadamente energéticas. Além disso, a maior proporção de gordura visceral, em animais de aptidão leiteira, resultaria, na prática, em maiores exigências de energia para mantença, em virtude da maior atividade metabólica do tecido adiposo interno em relação ao tecido adiposo periférico (THOMPSON et al., 1983; SOLIS et al., 1988).

\section{Conclusões}

Os pesos de omaso, abomaso, fígado, pulmão, coração, baço e rins, em valores absolutos, para os animais abatidos aos $190 \mathrm{~kg}$ de peso vivo, foram influenciados pelos níveis de concentrado nas dietas.

O comprimento do ID, em \% do PCVZ, dos animais abatidos aos $300 \mathrm{~kg}$ de peso vivo diminuiu linearmente com adição de concentrado nas dietas.

Os pesos do fígado e dos rins, em \% do PCVZ, dos animais abatidos dos $190 \mathrm{~kg}$ de peso vivo elevaram-se linearmente com o aumento da proporção de concentrado nas dietas.

O peso da gordura mesentérica, em valores absolutos e \% PCVZ, para os animais abatidos aos $300 \mathrm{~kg}$ de peso vivo, aumentou linearmente, com adição de concentrado nas dietas.

\section{Referências Bibliográficas}

ARAÚJO, G.G.L., SIGNORETTI, R.D., COELHO DA SILVA, J.F. et al. Comportamento biométrico do trato gastrointestinal e tamanho de órgãos internos de bezerros mestiços (Holandês $\mathrm{x}$ Zebu). Grupo 1. In: REUNIÃO ANUAL DA SOCIEDADE BRASILEIRA DE ZOOTECNIA, 33, Fortaleza, CE. 1996. Anais... Fortaleza: SBZ, 1996. p.396-398.

BERG, R.T., BUTTERFIELD, R.M. 1976. New concepts of cattle growth. New York: Sydney University. 240p.

CALIL, R.M. Inspeção “Ante Mortem”. In: CURSO INTERNACIONAL SOBRE TECNOLOGIA DA CARNE. Campinas, ITAL. p.41, 1978.

DROUILLARD, J.S., KLOPFENSTEIN, T.J., BRITTON, R.A. et al. 1991. Growth, body composition, and visceral organ mass and metabolism in lambs during and after metabolizable protein or net energy restritions. J. Anim. Sci., 69:3357-3375.
EUCLYDES, R.F. 1983. Manual de utilização do programa SAEG (Sistema para Análises Estatísticas e Genéticas). Viçosa - MG, 59p.

FERREL, C.L., GARRETT, W.N., HINMAN, N. 1976. Estimation of body composition in pregnant and non pregnant heifers. J. Anim. Sci., 42(5):1158-1166.

FERREL, C.L., JENKINS, T.G. 1983. Energy utilization by mature, nonpregnant, nonlactanting cows of different types. J. Anim. Sci., 58(1):234.

GALVÃO, J.G.C., FONTES, C.A.A., PIRES, C.C. et al. 1991. Características e composição física da carcaça de bovinos não-castrados, abatidos em três estágios de maturidade (Estudo II) de três grupos raciais. R. Soc. Bras. Zootec., 20(5):502-512.

GEAY, Y. Live weight measurement. In: EEC SEMINAR ON CRITERIA AND METHODS FOR ASSESSMENT OF CARCASS AND MEAT CHARACTERISTICS IN BEEF PRODUCTION EXPERIMENTS, 1975, Zeist. Proceedings... s.n.t.p.35-42, 1975.

HOGG, B.W. 1991. Compensatory growth in ruminants. In: PEARSON, A. M., DUTSUN, T. R. (Ed.) Growth regulation in farm animals - Advance in meat science research. v. 7, London: Elsevier Applied Science. p.105

JOHNSON, D.E., JOHNSON, K.A., BALDWIN, R.L. 1990. Changes in liver and gastrointestinal tract energy demands in response to physiological workload in ruminants. $J$. Nut., 120:649-655.

JONES, S.D.M., ROMPALA, R.E., JEREMIAH, L.E. 1985. Growth and composition of the empty body in steers of different maturity types fed concentrate or forage diets. $J$. Anim. Sci., 60(2):427-433.

JORGE, A.M., FONTES, C.A.A., PAULINO, M.F. Composição física e outras características quantitativas de carcaças de zebuínos não-castrados de quatro raças, abatidos em três estágios de maturidade. In: REUNIÃO ANUAL DA SOCIEDADE BRASILEIRA DE ZOOTECNIA, 33, Fortaleza, CE. Anais... Fortaleza: SBZ, 1996, p.188-189.

LUNT, D.K., BYERS, F.M., GREENE, L.W. et al. 1986. Effects of breed, diet and growth rate on vital organ mass in growing and finishing beef steers. J. Anim. Sci., 63(1):70-71. Supplement, 1 .

NATIONAL RESEARCH COUNCIL - NRC. 1989. Nutrient requirements of dairy cattle. 6.ed. Washington, D.C.157p.

OLIVEIRA, R.F.M., FONTES, C.A.A., CARNEIRO, L.H.D.M. et al. 1992. Biometria do trato gastrointestinal de bovinos de três grupos genéticos. R. Soc. Bras. Zootec., 21(2):205-210.

PERON, A.J., FONTES, C.A., LANA, R.P. et al. 1993. Tamanho de órgãos internos e distribuição da gordura corporal em novilhos de cinco grupos genéticos submetidos à alimentação restrita e "ad libitum". R. Soc. Bras. Zootec., 22(5):813-819.

RICHMOUD, C.E., LUNT, D.K., GREENE, L.W. et al. 1988. Effects of dietary restriction and subsequent re-alimentation on liver mass in growing/finishing beef steers. Nut. Reports Intern., 38:501-509.

SOLIS, J.C., BYERS, F.M., SCHELLING, G.T. et al. 1988. Maintenance requirements and energetic efficiency of cows of different breed types. J. Anim. Sci., 66:764.

THOMPSON, W.R., MEISKE, J.C. GOODRICH, R.D. et al. 1983. Influence of body composition on energy requirements of beef cows during winter. J. Anim. Sci., 56(5):1241-1251.

VALADARES FILHO, S.C. Digestão total e parcial da matéria seca e carboidratos em bovinos e bubalinos. Viçosa, MG:UFV, 1985, 148p. Tese (Doutorado em Zootecnia) - Universidade Federal de Viçosa, 1985.

Recebido em: 27/08/98 Aceito em: 25/01/99 